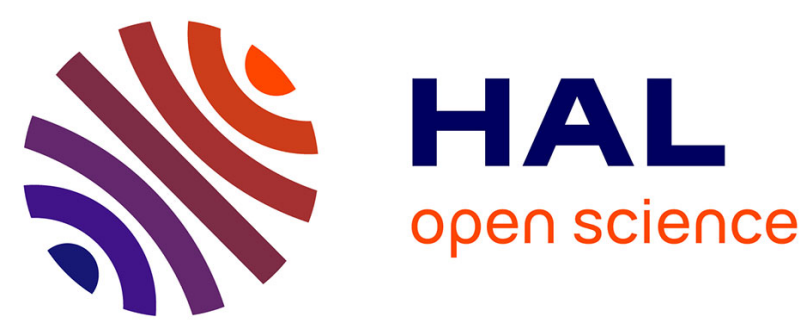

\title{
Influence du matériau de couverture sur les échanges énergétiques d'une serre: étude comparative verre normal - verre à faible émissivité. I. Influence sur les déperditions thermiques
}

\author{
Maria Baille, Jean-Claude Laury, Alain Baille, Gilbert Sappe
}

\section{To cite this version:}

Maria Baille, Jean-Claude Laury, Alain Baille, Gilbert Sappe. Influence du matériau de couverture sur les échanges énergétiques d'une serre: étude comparative verre normal - verre à faible émissivité.

I. Influence sur les déperditions thermiques. Agronomie, 1983, 3 (3), pp.197-202. hal-00884500

\author{
HAL Id: hal-00884500 \\ https://hal.science/hal-00884500
}

Submitted on 1 Jan 1983

HAL is a multi-disciplinary open access archive for the deposit and dissemination of scientific research documents, whether they are published or not. The documents may come from teaching and research institutions in France or abroad, or from public or private research centers.
L'archive ouverte pluridisciplinaire HAL, est destinée au dépôt et à la diffusion de documents scientifiques de niveau recherche, publiés ou non, émanant des établissements d'enseignement et de recherche français ou étrangers, des laboratoires publics ou privés. 


\title{
Influence du matériau de couverture sur les échanges énergétiques d'une serre: étude comparative verre normal - verre à faible émissivité. I. Influence sur les déperditions thermiques
}

\author{
Maria BAILLE, Jean-Claude LAURY \& Alain BAILLE $\left({ }^{*}\right)$ \\ avec la collaboration technique de Gilbert SAPPE $\left({ }^{*}\right)$ \\ Institut technique interprofessionnel de l'Horticulture, Station de Val de Loire, F 49000 Angers \\ (*) I.N.R.A., Station de Bioclimatologie, Centre de Recherches d'Avignon, F 84140 Montfavet
}

RÉSUMÉ

\begin{abstract}
Deux matériaux de couverture de serre ont été comparés : le verre horticole ordinaire et le verre à faible émissivité. L'analyse des différents types de déperdition thermique a été réalisće et montre que le verre à faible émissivité permet de réduire de façon significative les pertes par rayonnement $(70$ p. 100 en moyenne). Au niveau des pertes globales de la serre, les réductions dues au verre à faible émissivité varient, suivant les conditions climatiques, de 0 p. 100 (pluie) à 40 p. 100 (ciel clair, vent faible).

Sur 2 saisons entières de chauffage, l'économie apportée par le verre à faible émissivité a été en moyenne de 22 p. 100. Ce résultat est valable pour les conditions climatiques du Val de Loire ou pour des climats similaires.
\end{abstract}

Mots clés additionnels : Couverture, bilan d'énergie, pertes thermiques.

Influence of the cover material on the energy balance of a glasshouse : comparison of ordinary glass with low emissivity glass. I. Effect on heat loss

Two glasshouse cover materials were compared: standard horticultural glass and low emissivity glass. Analysis of the different heat loss types showed that low emissivity glass significantly reduced radiative losses ( $70 \%$ on average). The reduction in total heat losses depended on climatic conditions, varying from $0 \%$ (rain) to $40 \%$ (clear sky, low wind speed).

For two years of heating, the mean value of energy saving due to low emissivity glass would be $22 \%$. This result is valid for climatic conditions such as those in the Loire valley.

Additional key words : Cover, energy balance, heat loss.

\section{INTRODUCTION}

Le coût croissant de l'énergie d'origine fossile a entraîné, dans les cultures sous serre, la recherche de solutions permettant de réduire les déperditions thermiques des serres: l'utilisation de matériaux de couverture possédant des propriétés optiques particulières dans l'infrarouge thermique et, notamment, du verre à faible émissivité, est l'une de ces solutions.

Comme son nom l'indique, le verre à faible émissivité (V.F.E.) est un matériau verrier possédant un coefficient d'émissivité dans l'infrarouge lointain $(\lambda>3000 \mathrm{~nm})$ plus faible que celui du verre ordinaire. Cette propriété optique lui cst conférée grâce à la présence, sur l'une de ses faces, d'une mince couche d'oxyde d'étain. Dans la pratique, cette face est dirigée vers l'extérieur et l'autre face, qui se comporte comme le verre ordinaire, est dirigée vers l'intérieur. Le rôle principal de ce matériau est de diminuer de façon significative les pertes d'origine radiative au niveau de la toiture.

L'utilisation d'un tel matériau va entraîner un certain nombre de conséquences, non seulement au niveau des déperditions thermiques de la serre, mais également au niveau du microclimat interne, nocturne et diurne. Pour préciser ces différents points, une série de comparaisons entre deux serres identiques, l'une couverte en V.F.E. et l'autre en verre ordinaire a été réalisée. On présente dans cet article la partie concernant les déperditions thermiques et consommations en chauffage des 2 types de serre. Une $2^{c}$ partic traitera plus spécialement des effets sur les microclimats internes résultants (diurnes et nocturnes). 


\section{ESTIMATION THÉORIQUE DES PERTES RADIATIVES D'UNE SERRE}

Une approche théorique simple a été utilisée pour essayer d'estimer les pertes par rayonnement au niveau de la toiture de la serre, en fonction des propriétés optiques du matériau de couverture. Pour cela, on a explicité les bilans radiatifs au niveau des faces interne et externe de la paroi de la serre en fonction des propriétés optiques de la paroi, à savoir :

$-\varepsilon_{\mathrm{c}}$ et $\varepsilon_{\mathrm{i}}$, coefficients respectifs d'émissivité des faces externe (indice $\langle\mathrm{e} »$ ) et interne (indice $\langle\mathrm{i} »$ ) ;

$-\rho_{\mathrm{c}}$ et $\rho_{\mathrm{i}}$, coefficients de réflexion des faces externe et interne ;

$-\tau_{p}$, coefficient de transmission de la paroi.

$\mathrm{Si}$ on se reporte à la figure 1 , on peut expliciter $\mathrm{R}_{\mathrm{N}, \mathrm{i}}$, bilan radiatif de la face interne, et $R_{N . e}$, bilan radiatif de la face externe par (Bouchet \& Damagnez, 1967; Seginer \& LEVAV, 1970) :

$$
\begin{aligned}
& \mathbf{R}_{N, i}=R_{S}\left(1-\rho_{i}\right)-\tau \cdot R_{A}-\varepsilon_{i} \cdot \sigma \cdot T_{p}^{4}, i \\
& R_{N, e}=R_{A}\left(1-\rho_{e}\right)-\tau \cdot R_{S}-\varepsilon_{\mathrm{e}} \cdot \sigma \cdot T_{p}^{4}, e
\end{aligned}
$$

$R_{S}$ est le rayonnement de la surface du sol de la serre et $R_{A}$ le rayonnement de l'atmosphère. Ces termes peuvent s'écrire, en supposant que la surface de la serre et l'atmosphère rayonnent comme des corps noirs:

$$
\begin{aligned}
& \mathrm{R}_{\mathrm{A}}=\sigma \cdot \mathrm{T}_{\mathrm{A}}^{4} \\
& \mathrm{R}_{\mathrm{S}}=\sigma \cdot \mathrm{T}_{\mathrm{S}}^{4}
\end{aligned}
$$

$T_{p, e}$ et $T_{p . i}$ sont les températures des faces externe et interne de la paroi, $\mathrm{T}_{\mathrm{A}}$ la températurc apparente de l'atmosphère et $T_{S}$ la température de surface de la serre.

En tenant comptc de la relation $\rho+\tau+\varepsilon=1$, les relations (1) et (1 bis) s'écrivent :

$$
\begin{aligned}
& R_{N, i}=\tau\left(\sigma . T_{S}^{4}-\sigma \cdot T_{A}^{4}\right)+\varepsilon_{i}\left(\sigma \cdot T_{S}^{4}-\sigma \cdot T_{p, i}^{4}\right) \\
& R_{N, e}=\tau\left(\sigma . T_{A}^{4}-\sigma \cdot T_{S}^{4}\right)+\varepsilon_{v}\left(\sigma \cdot T_{A}^{4}-\sigma \cdot T_{p, e}^{4}\right)
\end{aligned}
$$

Dans les situations de vent nul ou faible, on peut considérer que les échanges par rayonnement sont prépondérants. En négligeant les termes convectifs, le bilan énergétique de la paroi se réduit alors à l'expression :

$$
\mathbf{R}_{\mathrm{N} . \mathrm{i}}+\mathrm{R}_{\mathrm{N} . \mathrm{e}}=0
$$

D'où :

$$
\varepsilon_{\mathrm{e}}\left(\sigma . \mathrm{T}_{\mathrm{A}}^{4}-\sigma . \mathrm{T}_{\mathrm{p}, \mathrm{e}}^{4}\right)+\varepsilon_{\mathrm{i}}\left(\sigma . \mathrm{T}_{\mathrm{S}}^{4}-\sigma . \mathrm{T}_{\mathrm{p}, \mathrm{i}}^{4}\right)=0
$$
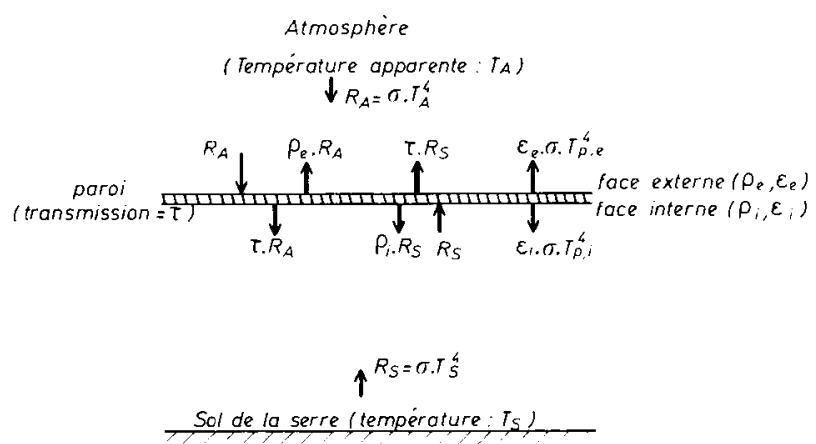

Figure 1

Bilan radiatif des faces interne et externe de la paroi.

Radiative balance of inner and outer side wall.
Les températures $T_{p . i}$ et $T_{p, i}$ sont relativement proches. On peut, en première approximation, poser : $T_{p, c}=T_{p, i}=T_{p}$. D'où :

$$
\sigma . \mathrm{T}_{\mathrm{p}}^{4}=\sigma \frac{\varepsilon_{\mathrm{e}} \cdot \mathrm{T}_{\mathrm{A}}^{4}+\varepsilon_{\mathrm{i}} \cdot \mathrm{T}_{\mathrm{S}}^{4}}{\varepsilon_{\mathrm{e}}+\varepsilon_{\mathrm{i}}} .
$$

Si on remplace $\sigma . T_{p}^{4}$ par la relation (6) dans l'expression de $R_{N . c}$, terme qui représente les échanges radiatifs avec l'extérieur, on aboutit à :

$$
\mathbf{R}_{\mathrm{N}, \mathrm{e}}=\left(\sigma \cdot \mathbf{T}_{\mathrm{A}}^{4}-\sigma \cdot \mathrm{T}_{\mathrm{S}}^{4}\right)\left(\tau+\frac{\varepsilon_{\mathrm{e}} \cdot \varepsilon_{\mathrm{i}}}{\varepsilon_{\mathrm{e}}+\varepsilon_{\mathrm{i}}}\right) .
$$

On constate donc que les pertes radiatives dépendent en premier licu :

- de l'écart de température $T_{A}-T_{S}$ entre l'atmosphère et le sol de la serre;

- des proprićtés optiques de la paroi dans l'infrarouge, $\varepsilon_{\mathrm{c}}, \varepsilon_{\mathrm{i}}$ et $\tau$.

La figure 2 présente la comparaison des valeurs de $R_{N, c}$ calculées en fonction de $\mathrm{T}_{\mathrm{A}}$, pour $\mathrm{T}_{\mathrm{S}}=20^{\circ} \mathrm{C}$, dans le cas d'un verre ordinaire $\left(\tau \simeq 0, \varepsilon_{\mathrm{c}}=\varepsilon_{\mathrm{i}}=0,9\right)$ et dans le cas d'un verre à faible émissivité $\left(\tau \simeq 0, \varepsilon_{\mathrm{c}}=0,25, \varepsilon_{\mathrm{i}}=0,90\right)$.

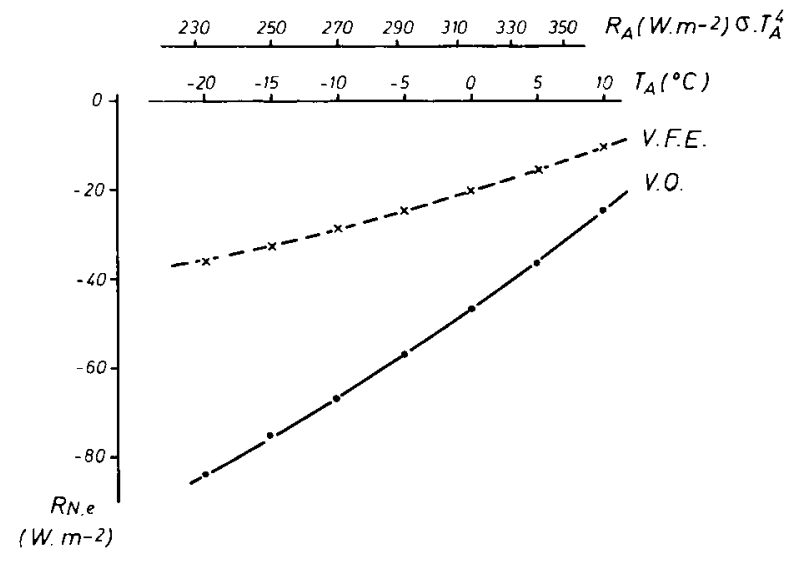

Figure 2

Valeurs calculées de $R_{N, e}$ en fonction de $T_{A}$, pour $T_{S}=20^{\circ} \mathrm{C}$, pour un verre ordinaire el pour un verre à faible émissivité.

Calculated values of $R_{N . e} v s$. $T_{A}$, for $T_{S}=20^{\circ} \mathrm{C}$, for an ordinary glass and a low emissivity glass.

\section{RÉSULTATS EXPÉRIMENTAUX}

\section{A. Installations et moyens expérimentaux}

\section{Les serres}

Les 2 éléments de serre choisis pour cette étude sont enclavés au milieu d'un bloc de serres expérimentales de la Station du Val de Loire (Institut Technique Interprofessionnel de l'Horticulture), à Angers. L'une des serres est couverte de verre ordinaire et l'autre de verre à faible émissivité. Les caractéristiques géométriques de ces éléments sont exprimées dans le tableau 1.

Le chauffage est assuré par thermosiphon, au moyen de tuyaux de circulation d'eau chaude répartis sous les tablettes, le long du pignon et à hauteur des cheneaux.

La température de consigne (température d'air prise à $1,20 \mathrm{~m}$ ) a étć fixée à $20^{\circ} \mathrm{C}$ pendant toute la durée des essais. 
TABLEAU 1

Caractéristiques géométriques des 2 serres choisies. Geometrical characteristics of the two glasshouses.

\begin{tabular}{|c|c|c|}
\hline & Scrre V.O. & Serre V.F.E. \\
\hline Longueur & $18,2 \mathrm{~m}$ & $16,2 \mathrm{~m}$ \\
\hline Largeur ....... & $9,0 \mathrm{~m}$ & $9,0 \mathrm{~m}$ \\
\hline Hauteur sous cheneaux ..... & $2,5 \mathrm{~m}$ & $2,8 \mathrm{~m}$ \\
\hline Hauteur sous faîtière... & $4,5 \mathrm{~m}$ & $4,8 \mathrm{~m}$ \\
\hline Surface du toit ................. & $180,0 \mathrm{~m}^{2}$ & $180,0 \mathrm{~m}^{2}$ \\
\hline Surface du pignon $\ldots \ldots \ldots \ldots$ & $31,5 \mathrm{~m}^{2}$ & $34,5 \mathrm{~m}^{2}$ \\
\hline 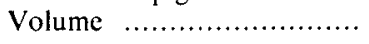 & $576,0 \mathrm{~m}^{3}$ & $600,0 \mathrm{~m}^{3}$ \\
\hline
\end{tabular}

Les mêmes cultures de plantes en pot ont été conduites sous les 2 éléments de serre (plantes ornementales posées sur tablettes, du type ficus, dieffenbachia, ...).

\section{Les mesures}

Un certain nombre de relevés ont été effectués en continu, sous serre et à l'extérieur. On a mesuré :

\section{a) Sous serre:}

- la puissance dissipée, $Q_{T}$, au moyen de compteurs de calorics :

- les températures des faces internes, $T_{p, i}$, et externe, $\mathrm{T}_{\mathrm{p.c}}$, de la paroi ;

- la température de l'air, $\mathrm{T}_{\mathrm{a}, \mathrm{s}}$, sous abri ventilé ;

- la température en indice actinothermique, $T_{i, a}$.

\section{b) A l'extérieur:}

- le rayonnement atmosphérique, $\mathrm{R}_{\mathrm{A}}$, au moyen d'un pyrradiomètre différentiel (bilanmètre) équipé d'un cache (BAILlE \& KaTZ, 1981);

- la température de l'air extérieur, $\mathrm{T}_{\mathrm{a}, \mathrm{e}}$;

- la vitesse du vent, $V$, à $1 \mathrm{~m}$ environ au-dessus du niveau des faittières.

Toutes les mesures de température ont été réalisées avec des sondes-platinu, montées en pont de Wheatstone.

Les données sont enregistrées sur une acquisition de données à cassettes KLAR-TAD qui effectue l'intégration horaire des tensions délivrées par les capteurs. Les cassettes sont lues sur l'ordinateur MITRA-15 du Centre d'Avignon et les données, mises sur fichier, sont alors traitées par des programmes permettant de calculer les paramètres recherchés.

\section{B. Résultats}

\section{Pertes par rayonnement au niveau du toit et des pignons}

Les pertes radiatives par unité de surface $\left(\mathrm{W} . \mathrm{m}^{-2}\right)$ au niveau du toit, $R_{N . t}$, et des pignons, $R_{N, p}$, ont été calculées à partir des valeurs mesurées de $R_{A}$ et des températures des faces externes de paroi, $T_{p, c}$. (Les détails du calcul sont donnés en annexe). L'énergie totale dissipée par rayonnement est alors :

$$
\mathrm{Q}_{\mathrm{R}}=\mathrm{R}_{\mathrm{N} . \mathrm{t}} \cdot \mathrm{S}_{\mathrm{t}}+\mathrm{R}_{\mathrm{N} \cdot \mathrm{p}} \cdot \mathrm{S}_{\mathrm{p}}
$$

$S_{1}$ et $S_{p}$ étant respectivement les surfaces de la toiture et des pignons.

Les résultats, présentés sous forme de moyenne de $R_{N . t}$ entre $20 \mathrm{~h} 00$ et $04 \mathrm{~h} 00$, sont donnés sur la figure 3 , pour la période octobre 1978-avril 1979. On voit que:

- pour lc verre ordinaire, les pertes par rayonnement au

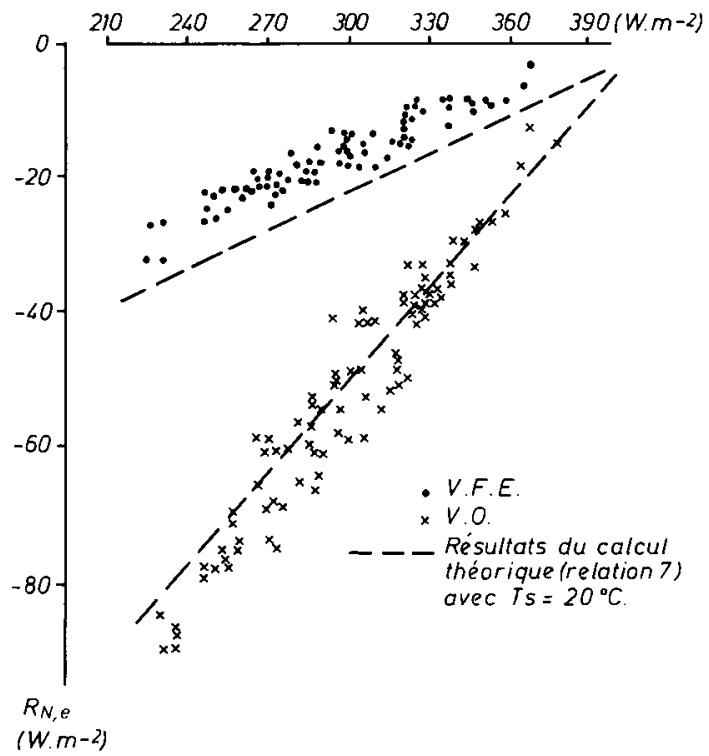

Figure 3

Pertes par rayonnement au niveau de la toiture, $R_{N, e}$ en fonction $d u$ rayonnement atmosphérique (période octobre 1978-avril 1979).

Radiative loss at roof level, $R_{N . e} v s$. atmospheric radiation (period October 1978-April 1979).

niveau de la toiture $R_{\mathrm{N}, \mathrm{t}}$ varient de $30 \mathrm{~W} . \mathrm{m}^{-2}$ par ciel couvert $\left(\mathrm{R}_{\mathrm{A}} \simeq 320\right.$ à $\left.340 \mathrm{~W} \cdot \mathrm{m}^{-2}\right)$ à $80 \mathrm{~W} \cdot \mathrm{m}^{-2}$ par ciel dégagé $\left(R_{A} \simeq 240 \mathrm{~W} \cdot \mathrm{m}^{-2}\right)$;

- pour lc V.F.E., ces pertes varient respectivement de $10 \mathrm{~W} \cdot \mathrm{m}^{-2}$ par ciel couvert à $25 \mathrm{~W} \cdot \mathrm{m}^{-2}$ par ciel clair.

La réduction des pertes par rayonnement due à la couverture en V.F.E. varie donc entre 65 p. 100 et 75 p. 100 , ce qui confirme les estimations de l'approche théorique précédemment décrite.

Si on tient compte également des pertes par rayonnement des pignons, $R_{N, p}$, on constate que les pertes par rayonnement $Q_{R}$, rapportées aux déperditions globales $Q_{T}$ (mesurées par les compteurs de calories), varient de $30 \mathrm{p} .100$ à $70 \mathrm{p} .100$ pour le verre ordinaire et de $10 \mathrm{p} .100$ à 30 p. 100 pour le verre à faible émissivité, cela pour des conditions de vent faible ou modéré $\left(V \leqslant 2 \mathrm{~m} . \mathrm{s}^{-1}\right)$. Sur l'échantillon considéré, le poids moyen des pertes par rayonnement par rapport aux pertes totales est de 45 p. 100 dans le cas du verre ordinaire et de 22 p. 100 dans le cas du V.F.E.

\section{Pertes par convection et par fuites}

Les déperditions globales de la serre, $\mathrm{Q}_{\mathrm{T}}$, sont la somme des déperditions par rayonnement, $Q_{R}$, par conductionconvection, $Q_{c c}$, par fuites, $Q_{f}$, et par conduction dans le sol, Q.

$$
\mathrm{Q}_{\mathrm{T}}=\mathrm{Q}_{\mathrm{R}}+\mathrm{Q}_{\mathrm{cc}}+\mathrm{Q}_{\mathrm{f}}+\mathrm{Q}_{\mathrm{s}}
$$

$\mathrm{Q}$, est en général négligeable devant les autres termes. Connaissant $Q_{T}$ et $Q_{R}$, on peut donc déduire les valeurs $\mathrm{Q}_{\mathrm{cc}}+\mathrm{Q}_{\mathrm{f}}$. On constate que le niveau de ces pertes est légèrement supérieur dans le cas du V.F.E. (fig. 4), de l'ordre de 10 p. 100 , ce que l'on peut sans doute attribuer aux températures de paroi qui sont plus élevées sous la serre V.F.E. (Cf. article suivant).

Le poids relatif des pertes par rayonnement $\left(Q_{R}\right)$ par rapport aux pertes par convection et par fuites $\left(\mathrm{Q}_{\mathrm{cc}}+\mathrm{Q}_{f}\right)$ est présenté sur les figures 5 (vent $<2,0 \mathrm{~m} . \mathrm{s}^{-1}$ ) ct 6 (vent $>2,0 \mathrm{~m} \cdot \mathrm{s}^{-1}$ ) $\mathrm{cn}$ fonction de la différence entre la 


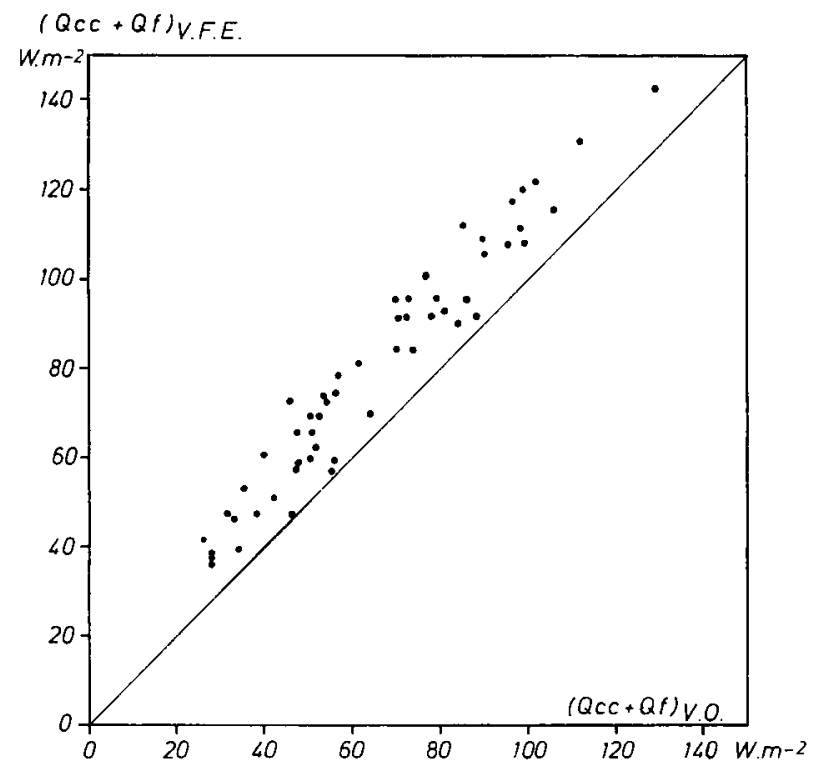

Figure 4

Comparaison des pertes par conduction-convection et fuites, $Q_{\text {ci }}+Q_{p}$, dans les 2 serres.

Comparison of conduction-convection and ventilation losses, $Q_{c}+Q_{\beta}$ in the two glasshouses.

$Q_{R} / Q_{c C}+Q_{f}$

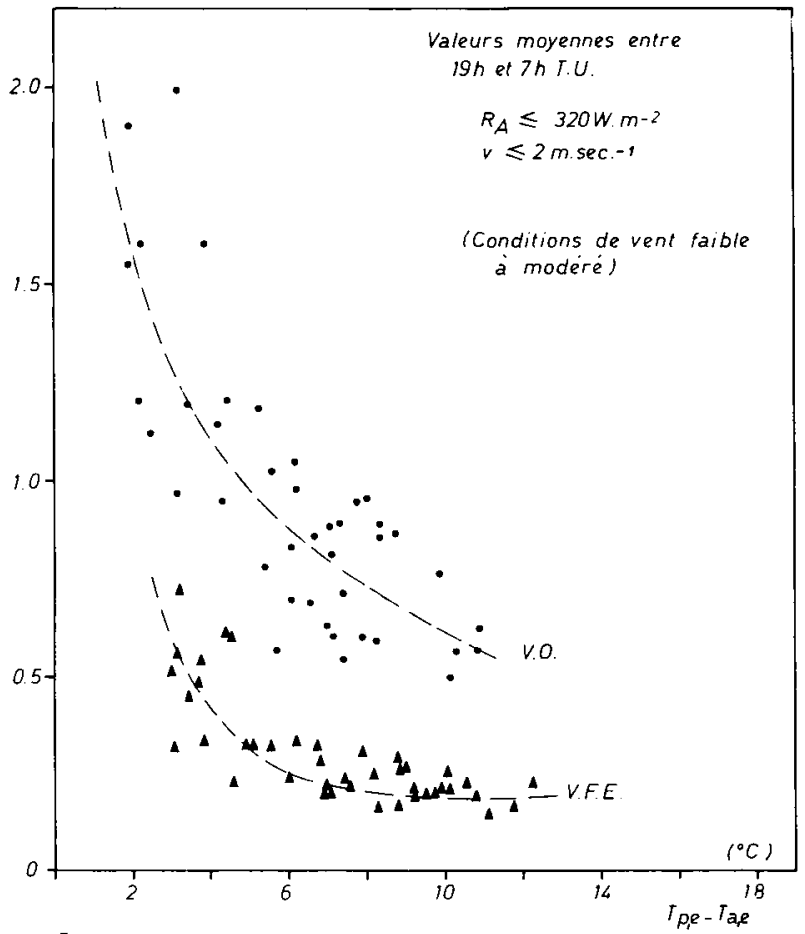

Figurc 5 Rapport $\frac{Q_{R}}{Q_{11}+Q_{1}}$ en fonction de l'écart $T_{p, e}-T_{a, e}$ pour
$V<2 m . s^{-1}$.

Ratio $\frac{Q_{k}}{Q_{c c}+Q_{f}} v s .\left(T_{p}-T_{a, e}\right)$ for $V<2 m \cdot s^{-1}$.

température de paroi, $T_{p, e}$, et la température de l'air extérieur, $\mathrm{T}_{\mathrm{a} . \mathrm{c}}$. Dans le $1^{\mathrm{cr}}$ cas, on constate que ce rapport varie :

- entre 0,50 et 2,0 pour le verre ordinaire ;

- entre 0,20 et 0,60 pour le verre à faible émissivité.

Dans le $2^{\mathrm{C}}$ cas, où la dispersion des points expérimentaux est plus importante, le rapport est à peu près constant pour

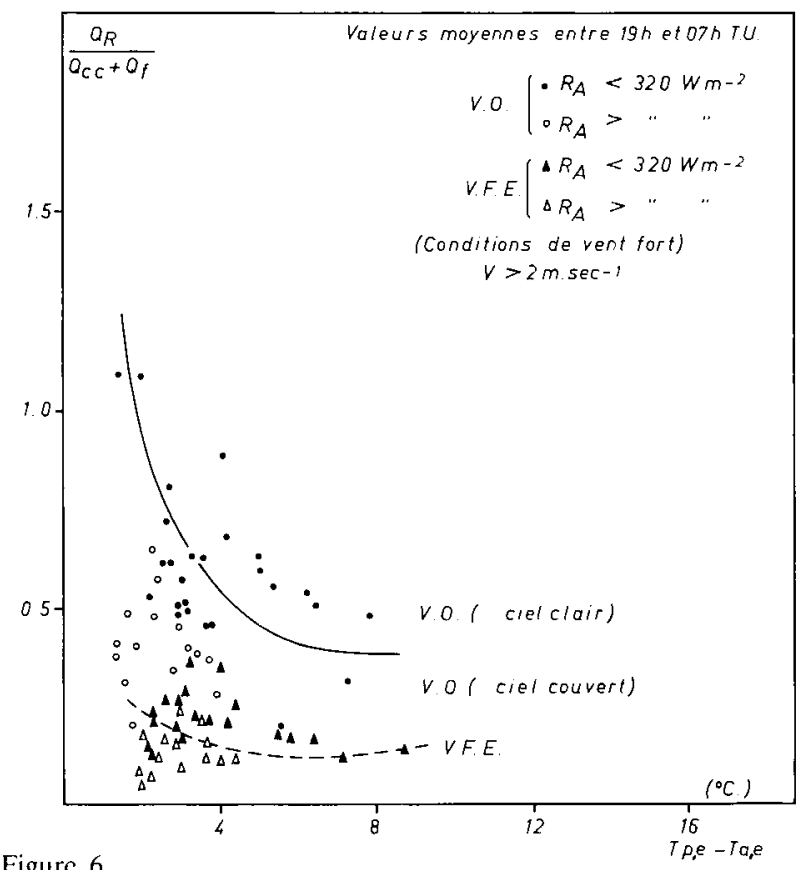

Figurc 6

Rapport
$V>2 m$
$\frac{Q_{R}+l}{s^{-l}}+Q_{f}$ en fonction de l'écart $T_{p . e}-T_{a . e}$ pour Ratio $\frac{Q_{R}}{Q_{c c}+Q_{f}} v s .\left(T_{p, e}-T_{a, e}\right)$ for $V>2 \mathrm{~m} \cdot \mathrm{s}^{-1}$.

le verre à faible émissivité $(\simeq 0,20)$ alors qu'il varie de 0,30 à 1,00 pour le verre ordinaire.

Les pertes par rayonnement, qui peuvent atteindre le double des pertes par convection et par fuites dans le cas du verre ordinaire, ne dépassent en aucun cas 60 p. 100 de ces pertes dans le cas du verre à faible émissivité.

A partir de ces données, et sachant que le verre à faible émissivité n'agit principalement que sur les pertes par rayonnement en les réduisant de $70 \mathrm{p} .100$, on peut déjà déduire les ordres de grandeur des économies de chauffage qu'on peut attendre de ce matériau:

a) par conditions de vent faible, $\mathrm{Q}_{\mathrm{R}}$ représente 35 à 65 p. 100 des pertes totales d'une serre en verre ordinaire : la réduction apportée par le verre à faible émissivité variera donc entre 25 et 45 p. 100 ;

b) par vent fort, $Q_{R}$ ne représente que 10 à 40 p. 100 des pertes totales d'une serre en verre ordinaire : la réduction apportée par le verre à faible émissivité sera donc comprise dans la fourchette $5-30$ p. 100.

\section{Coefficients globaux de déperdition thermique}

On définit en général le coefficient global de déperdition thermique de la serre, $\mathrm{K}$, par la relation :

$$
K=\frac{Q_{T}}{\left(S_{T}+S_{p}\right)\left(T_{a, s}-T_{a, e}\right)}
$$

$Q_{T}$ étant la puissance dissipée (en $\mathrm{W}$ ) et $S_{T}+S_{p}$ la surface de déperdition (en $\mathrm{m}^{2}$ ). $\mathrm{K}$ est exprimé en $\mathrm{W} \cdot \mathrm{m}^{-2} \cdot{ }^{\circ} \mathrm{C}^{-1}$.

Les 2 ćléments de serre étudiés ont des surfaces de pignon, $S_{p}$, et donc des volumes, légèrement différents. Une correction a été appliquée qui rapporte les résultats à des scrres ayant la même surface de déperdition et le même volume.

$\mathrm{Si}$ on porte les valeurs de $\mathrm{K}$ en fonction de la vitesse du vent (fig. 7), on obtient les relations classiques de variation 


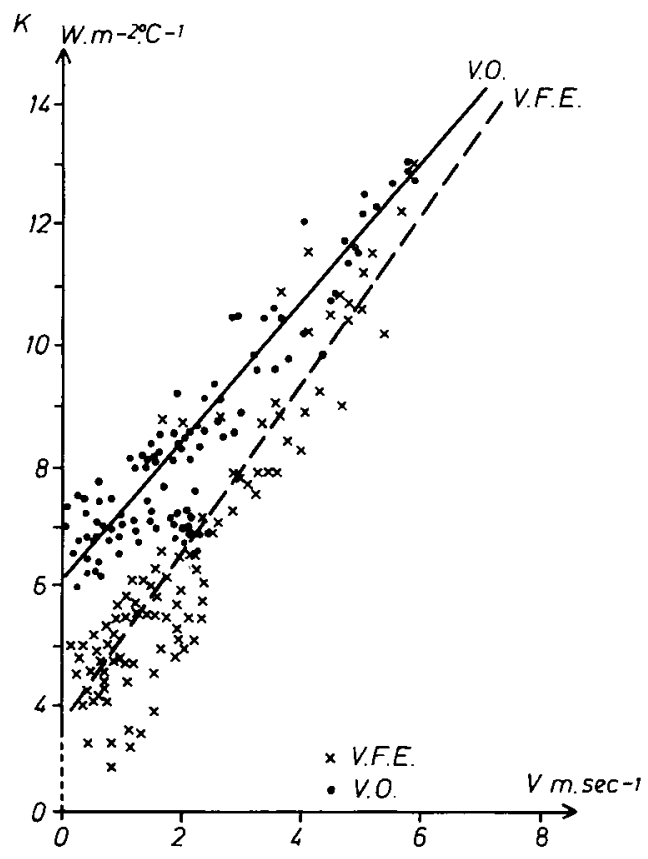

Figure 7

Variation du coefficient global de déperdition thermique, $K$, en fonction de la vitesse du vent, $V$, pour les 2 types de verre.

Variation of total heat loss coefficient, $K, v s$. wind speed, $V$, for the 2 glass covers.

linéaire de $\mathrm{K}$ en fonction de la vitesse du vent. Les valeurs de $\mathrm{K}$ pour le verre à faible émissivité sont inférieures à celles obtenues pour le verre ordinaire. Les différences les plus importantes se situent par vent nul ou modéré et atteignent
40 p. 100. Elles diminuent progressivement et ne sont que de 5 p. 100 pour $\mathrm{V}>6 \mathrm{~m} . \mathrm{s}^{-1}$.

$\mathrm{Si}$ l'on représente les variations de $\mathrm{K}$ en fonction du rapport $Q_{R} / Q_{c c}+Q_{f}$ (fig. 8), on constate que lorsque ce rapport augmente, $\mathrm{K}$ tend vers une valeur asymptotique égale à $6,5 \mathrm{~W} \cdot \mathrm{m}^{-2} \cdot{ }^{\circ} \mathrm{C}^{-1}$ dans le cas du verre ordinaire et à $3,5 \mathrm{~W}, \mathrm{~m}^{-2},{ }^{\circ} \mathrm{C}^{-1}$ dans le cas du verre à faible émissivité, c'est-à-dire les valeurs correspondant à $\mathrm{V}=0$. On observe également une dispersion des points expérimentaux beaucoup plus importante dans le cas du verre ordinaire, ce qui confirme la plus grande sensibilité du verre ordinaire aux pertes par rayonnement (HORIGUCHI, 1978).

\section{Consommation d'énergie des serres}

a) Rapport des consommations d'énergie, $Q_{V F E} / Q_{V O}$

Les valeurs moyennes journalières de ce rapport sont portées sur la figure 9 en fonction de la vitesse du vent. Ce rapport varie entre 0,65 et 0,95 , avec des valeurs très proches de 1,0 qui sont observées par conditions de pluie : une pellicule d'eau à la surface du verre à faible émissivité annule ses effets.

\section{b) Bilan sur la saison de chauffage}

Pour les 2 périodes de chauffage étudiées (octobre 1978 à avril 1979 et octobre 1979 à mars 1980), le bilan énergétique des 2 serres est résumé dans le tableau 2.

L'économic moyenne est donc de l'ordre de 22 p. 100 , avec une variabilité journalière importante comme on a pu le voir précédemment (fig. 9).

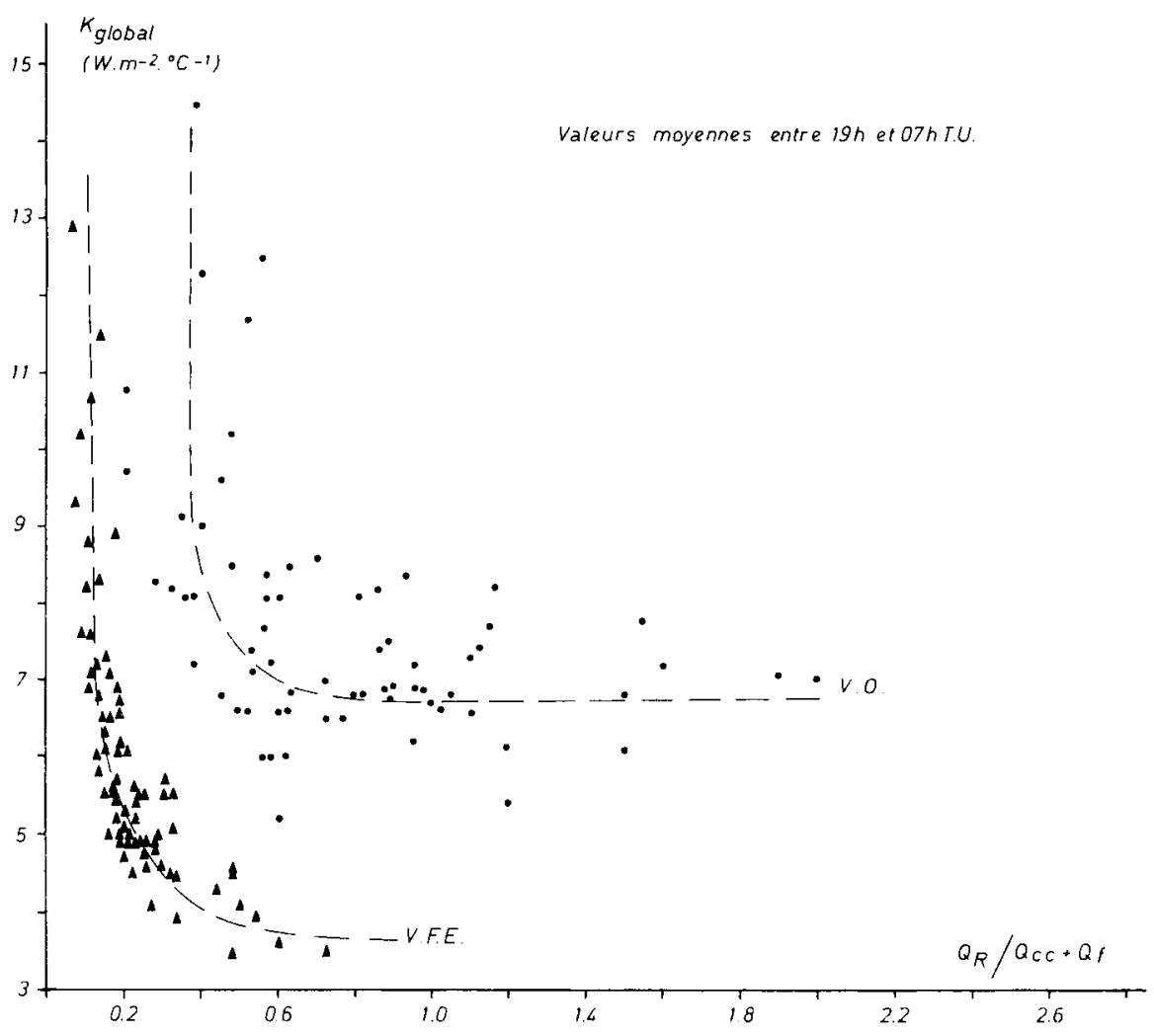




\section{TABLEAU 2}

Valeurs moyennes des rapports des consommations d'énergie et des rapports $\frac{Q_{K}}{\mathrm{Q}_{\mathrm{T}}}$ sur l'ensemble des saisons de chauffe 1978-79 et 1979-80.

Mean values of energy consumption and of ratios $\frac{Q_{R}}{\mathrm{Q}_{\mathrm{T}}}$ for the whole heating periods 1978-79 and 1979-80.

\begin{tabular}{|c|c|c|c|c|}
\hline & $\begin{array}{c}\text { Rapport } \frac{Q_{\mathrm{VIt}}}{\mathrm{Q}_{\mathrm{Vo}}} \\
\text { (p. 100) }\end{array}$ & $\begin{array}{c}\text { Economic d'énergie } \\
\text { due au verre à } \\
\text { faible émissivité } \\
\text { (p. 100) }\end{array}$ & $\begin{array}{l}\text { Rapport } \\
\text { Verre ordinaire }\end{array}$ & $\begin{array}{l}\frac{\mathrm{Q}_{\mathrm{R}}}{\mathrm{Q}_{\mathrm{T}}}(\mathrm{p} \cdot 100) \\
\quad \begin{array}{c}\text { Verre à faible } \\
\text { émissivité }\end{array}\end{array}$ \\
\hline $\mathrm{v} \leqslant 2 \cdot \mathrm{m} \cdot \mathrm{s}^{-1}$ & 72 & 28 & 46 & 22 \\
\hline $2<v \leqslant 4 \cdot m \cdot s^{-1}$ & 86 & 14 & 33 & 13 \\
\hline $\mathrm{V}>4 . \mathrm{m} . \mathrm{s}^{1}$ & 92 & 8 & 21 & 8 \\
\hline Moyenne & 78 & 22 & 40 & 18 \\
\hline
\end{tabular}

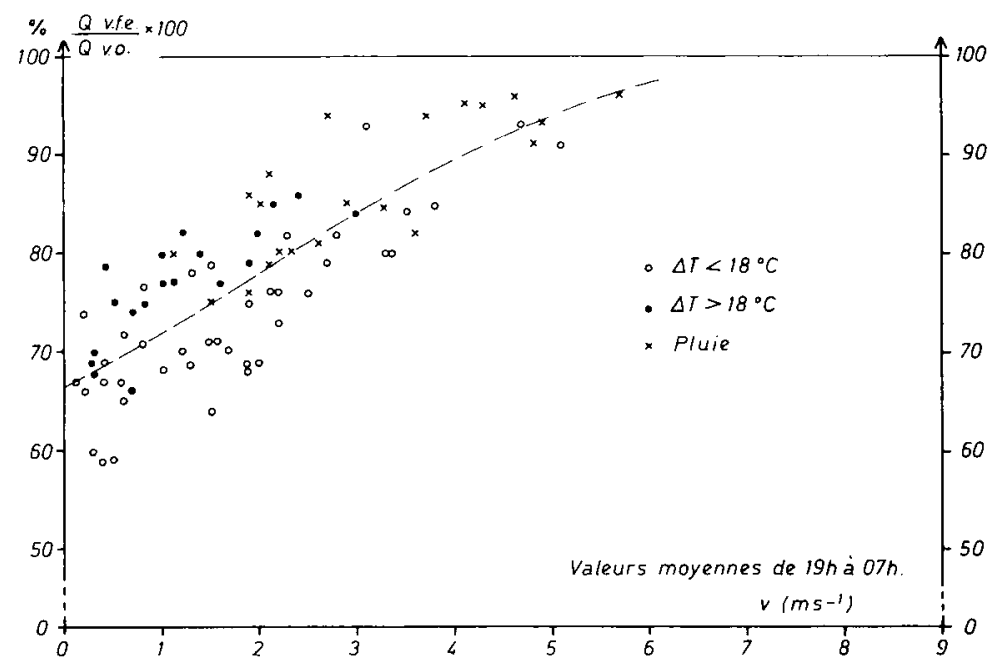

Figure 9

Evolution du rapport des consommations $\frac{Q_{V F E}}{Q_{V O}}$ en fonction de la Consumption ratio $\frac{Q_{i+k}}{Q_{v o}}$ vs. wind speed, $V$ vitesse du vent, $V$.

\section{CONCLUSION}

Le verre à faible émissivité est un matériau de couverture de serre qui permet de réduire de façon significative les pertes par rayonnement ( 70 p. 100 en moyenne). Le poids de ces pertes par rapport aux pertes globales de la serre étant très variable en fonction des conditions climatiques (nébulosité, vent), les ćconomies d'énergie induites par l'utilisation d'un tel matériau peuvent varier de quelques p. 100 (pluie, vent fort) à 40 p. 100 (ciel clair, vent faible).
La réduction des pertes globales de la serre estimée sur 2 saisons de chauffe successives est de 22 p. 100. Ce chiffre pcut varier légèrement en fonction :

- du climat de la région (nébulosité, vitesse du vent) ;

- de la température de consigne affichée dans la serre ;

- du systc̀me de chauffage adopté : les différents postes de déperdition thermique peuvent varier assez sensiblement suivant le type de chauffage utilisć (TANTAU, 1974).

Reçu le 15 juin 1982. Accepté le 28 octobre 1982.

\section{RÉFÉRENCES BIBLIOGRAPHIQUES}

Baille A., Katz M., 1981. Le point sur les méthodes et apparcils de mesure du rayonnement atmosphérique. Note interne M/81/4, I.N.R.A., Station de Bioclimatologie d'Avignon.

Bouchet R. J., Damagnez J., 1967. Etude théorique du bilan d'énergic des serres. 4' Congrès International du chauffage et de la climatisation, Paris, mai 1967.

Horiguchi I., 1978. The variation of heating load coefficient for a grecnhouse and overall heat transfer coefficient through plastic film. J. agric. Meteorol., 33 (4), 175-181.
Seginer I., Levav N., 1970. Models as tools in greenhouse climate design. Research Note 150-085, Technion Research and Development Foundation. Haïfa, Israc̈l.

Tantau H. J., 1974. Gartenbautechnische Informationen im Gewächshaüs Bercchnung Austegung Konstruktion. Institut für technik in gartenbau und landwirtschaft, Hannover. 